\title{
Enhancement of electroencephalogram activity in the theta-band range during unmatched olfactory-taste stimulation
}

\author{
Saori Maeda ${ }^{1,2} \cdot$ Hiroshi Yoshimura ${ }^{1}$
}

Received: 19 August 2018 / Accepted: 17 March 2019 / Published online: 4 April 2019

(c) The Physiological Society of Japan and Springer Japan KK, part of Springer Nature 2019

\begin{abstract}
The aim of this study was to investigate how odor stimulation affects taste perception. Electroencephalogram (EEG) signals were measured from the frontal region of the head in normal, healthy subjects, and frequency analyses were performed. Each odor stimulation was delivered while the subject was tasting chocolate, using chocolate paste as the odorant for 'matched odor stimulation,' and garlic paste for 'unmatched odor stimulation.' Differences in EEG signals appeared between the matched and unmatched arms of the study. Comparison of the frequencies of EEGs captured under the condition of unmatched odor stimulation with those captured under the condition of matched odor stimulation showed that the occupancy rate of the thetafrequency band under the condition of unmatched odor stimulation was higher than that under the condition of matched odor stimulation. Interestingly, a negative correlation existed between the occupancy rate of the theta-frequency band and the subjective feeling of chocolate sweetness. The present findings suggest that when humans receive odors that do not match with the foods being consumed, subjective feelings are disturbed and theta-band brain activity is increased while the unmatched information is cross-checked.
\end{abstract}

Keywords Electroencephalogram $\cdot$ Fast Fourier Transform analysis $\cdot$ Smell $\cdot$ Taste $\cdot$ Theta

\section{Introduction}

Taste and smell are fundamental senses by which humans obtain chemical information. Feelings of deliciousness when consuming foods can thus be produced by the perception of the chemical information extracted from the foods themselves. The molecules causing taste and smell bind to gustatory and olfactory receptors expressed on the tongue and olfactory epithelium, respectively. The resulting chemical signals are first conveyed to the primary gustatory and olfactory cortices, following which the information is processed at higher levels of the cortex [1-4]. The gustatory and olfactory cortices are strongly connected to the limbic system

Hiroshi Yoshimura

hyoshimu@tokushima-u.ac.jp

1 Department of Molecular Oral Physiology, Institute of Biomedical Sciences, The Tokushima University Graduate School, 3-18-15 Kuramoto, Tokushima 770-8504, Japan

2 Department of Oral Health Sciences, Faculty of Nursing and Health Care, BAIKA Women's University, 2-19-5, Shukunosho, Osaka, Ibaraki 567-8578, Japan and, accordingly, these chemical senses are also strongly connected with emotion [5,6]. The limbic system plays important roles in memory storage, retrieval, recognition and the production of various emotional feelings [2, 7-9]. The perceived deliciousness of food may therefore be partly based on memory and emotion with regard to the food in question, formed by personal experience.

Many foods contain specific substances that produce their own particular taste and odor. Food odor can increase the taste intensity of food $[10,11]$ by acting through crossmodal interactions $[12,13]$. Humans recognize foods by referring to the combination of taste and smell [14]. When the smell of a food differs from the smell that the food is usually associated with, we may experience difficulty in recognizing that food. In such cases, the processing of chemical information might be disturbed. However, the precise mechanisms involved in such disturbances remain unexplained.

Chocolate is a familiar food that is usually associated with feelings of pleasantness and an increased positive mood [15, 16]. In addition, chocolate can be easily identified by its specific combination of smell and taste. Therefore, in the present study we focused on chocolate as tastant. The deliciousness of chocolate experienced by the person consuming the 
chocolate may be enhanced or attenuated, depending on the associated smells. The frequency of electroencephalogram (EEG) signals is related to underlying activities of the brain; as such, EEG signals can be used to investigate perceptual, cognitive and emotional functions [17-21].

In the study reported here, we used Fast Fourier Transform (FFT) analysis to investigate how changes in the feelings associated with a taste correlate with brain activities when an unmatched odor stimulation is introduced into the association.

\section{Materials and methods}

All procedures performed in studies involving human participants were in accordance with the ethical standards of the institutional and/or national research committee and with the 1964 Declaration of Helsinki and its later amendments or comparable ethical standards. The institutional ethics committee at Tokushima University approved the study reported here (No. 2575-2]. Informed consent was obtained from all individual participants included in the study.

Nine healthy participants with a mean ( \pm standard deviation [SD]) age of $30.2 \pm 12.3$ years were enrolled in experiments using chocolate as a tastant, and another nine healthy participants with a mean age of $33.3 \pm 15.8$ years were included in control experiments in which no tastant was used. EEGs were recorded from the frontal region of the scalp (Fp1, Fp2) using an Alphatec IV EEG recorder [Brain Function Research \& Development Center [BFRDC], Osaka, Japan]. Frequency analyses of the EEGs were performed with a MinD Sensor V (BFRDC). During one session $(60 \mathrm{~s})$, data were collected every $1 \mathrm{~s}$. The sampling rate was $1024 \mathrm{~Hz}$. EEG epochs with artifacts were automatically identified, and artifact-free EEG epochs were used in the frequency analysis.

During the experiments, participants were seated in a body sonic chair (RFRESH1 Excellent, Tokyo, Japan) and listened to consonant music for relaxation. Milk chocolate (Morinaga \& Co., Tokyo, Japan) was used as a tastant. Chocolate paste (Morinaga \& Co.) was used as an odorant in the 'matched odor stimulation' arm, and garlic paste (House Food Corporation, Tokyo, Japan) was used as an odorant in the 'unmatched odor stimulation' arm. At the start of the session, the participant put a small piece of chocolate in his/her mouth and tasted it. For odor stimulation, the odorant was smeared onto cosmetic paper and placed close to the nose of the participant. EEGs were recorded for $60 \mathrm{~s}$ per session. After the session, the participant was asked to rate the perception of chocolate sweetness as a "chocolate sweetness score' on a scale of 1-5 ( 0 , no feeling of chocolate sweetness; +5 , extreme feeling of chocolate sweetness). As a reference for chocolate sweetness, participants were asked to consider the sweetness of chocolate with no odor stimulation as a chocolate sweetness score of 2.5. As a control experiment, odor stimulation with chocolate paste or garlic paste was delivered in the same way as mentioned above to participants without taste stimulation (no chocolate in the mouth), and EEGs were recorded for $60 \mathrm{~s}$ per session. As another control experiment, EEGs were recorded for $60 \mathrm{~s}$ per session while the participant was tasting chocolate in the absence of any odor stimulation.

When a small piece of chocolate is carried to the mouth, olfactory receptors detect the odor of the chocolate retronasally [22]. All participants were able to distinguish the odors of milk chocolate (retronasal) and chocolate paste (orthonasal) when the odors were presented separately. However, the retronasal pathway provides a smaller olfactory input than the orthonasal pathway [23, 24]. We therefore adopted cases using no odorants to represent the condition of no odor stimulation.

Individual EEG band frequencies were analyzed from 4 to $23 \mathrm{~Hz}$. The occupancy rate of each frequency was calculated as the power of each frequency divided by the summed power for all frequencies. The ratio was defined as the unit occupancy rate (\%). Activity in each frequency was first assessed by the unit occupancy rate per second. Then unit occupancy rates through one session (60 s) were averaged; these averaged data were used to determine the occupancy rate (\%) in this study. Details on EEG recordings and data analysis have been described previously by Yoshimura et al. [25].

\section{Results}

Data were collected from nine healthy participants in experiments in which chocolate was used as a tastant. In order to have a standard reference point, each participant was asked to rate the chocolate sweetness score of the tastant as 2.5 under the condition of tasting the chocolate without odor stimulation. The chocolate sweetness score under the condition of odor stimulation was then rated relative to that reference point of 2.5. Subjective feelings of chocolate sweetness were compared between conditions of unmatched odor stimulation and matched odor stimulation. For all participants, chocolate sweetness scores under the condition of matched odor stimulation were higher than the reference score. Conversely, chocolate sweetness scores under the condition of unmatched odor stimulation were lower than the reference score. Differences in chocolate sweetness scores between the two conditions were significant for all nine participants based on analyses using Origin8 software (OriginLab Co., Northampton, MA USA): $1.1 \pm 0.49$ (mean \pm standard deviation) (matched) vs. $3.9 \pm 0.33$ (unmatched); paired $t$ test, $p=4 \times 10^{-6}$ (Fig. 1). Interestingly, in addition to decreased 


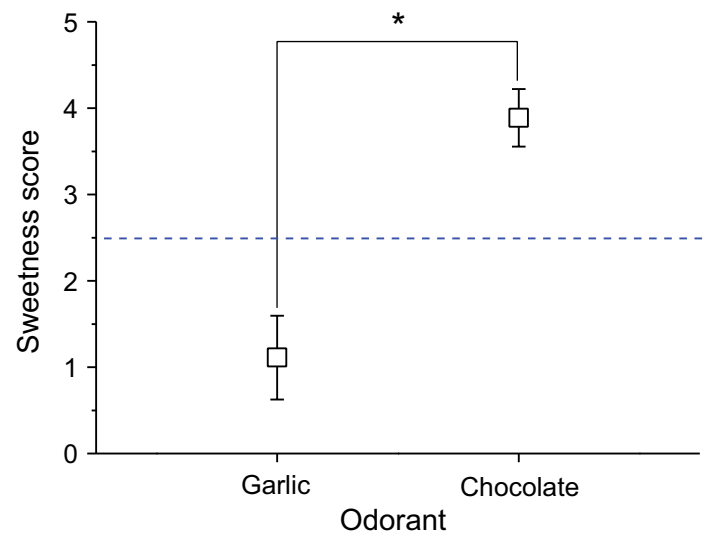

Fig. 1 Comparison of chocolate sweetness scores (scores of 1-5, where $0=$ no feeling of chocolate sweetness and $+5=$ extreme feeling of chocolate sweetness). Subjective feelings of chocolate sweetness under conditions of unmatched (garlic) and matched (chocolate) odor stimulation, respectively, while tasting chocolate. Sweetness scores were averaged and plotted (mean \pm standard deviation). Asterisk denotes a significant difference between chocolate sweetness scores and chocolate recognition scores

feelings of sweetness, participants showed a tendency to be unable to specifically discern what the food was under the condition of unmatched odor stimulation.

Objective differences between unmatched odor stimulation and matched odor stimulation conditions were apparent in the EEG signals collected from the nine healthy participants. Profiles of mean occupancy rates obtained from the frequency analysis are shown in Fig. 2a, b. Figure 2c shows the profiles of the two conditions superimposed. In the present study, we focused on theta, alpha and beta frequency bands. To assess the occupancy rate of the respective frequency bands, we calculated the areas surrounded by the lines of occupancy rates and the frequency axis $(\mathrm{Hz} \times \%)$ and termed the result the 'areal occupancy rate' (Fig. 2c). Mean areal occupancy rates of the respective frequency bands under conditions of unmatched and matched olfactory stimulations are shown in Fig. 2d. To investigate which frequency bands of EEG reflected different patterns of olfactory stimulation, we then subtracted the areal occupancy rates under the condition of unmatched odor stimulation from those under the condition of matched odor stimulation and compared the results. Mean differences for theta, alpha and beta bands were $5.25 \pm 4.48,-2.83 \pm 2.49$ and $-3.49 \pm 4.01(\operatorname{mean} \pm \mathrm{SD})$, respectively. One-way analysis of variance (ANOVA) using Origin8 software was used to evaluate the statistical significance of the 'areal difference values' in the three frequency bands. Statistical differences in areal difference values were identified $(F=15.1$; $P=5.7 \times 10^{-5}$ ). Scheffe's post hoc test was then used to detect significant differences between areal difference values in the respective frequency bands. These analyses revealed that differences in areal difference values between unmatched and matched odor stimulation conditions were significantly greater in the theta frequency band than in the other frequency bands (alpha: $F=10.4, P=5.5 \times 10^{-4}$; beta: $F=12.2, P=2.2 \times 10^{-4}$ ), suggesting that unmatched olfactory stimulation enhanced EEG activities in the theta frequency band.

Subjective differences (Fig. 1) and objective differences (Fig. 2) may be correlated. In other words, brain activities in the theta band might correlate with a disturbance in taste perception when unmatched odor stimulation was applied. Correlations between the chocolate sweetness score and areal occupancy rate in the theta, alpha and beta bands were therefore examined. In the theta frequency, a negative correlation was seen between the chocolate sweetness score and areal occupancy rate $\left(n=18, R^{2}=0.282, F=7.69\right.$, $P=0.01$; Fig. 3a). Correlations between the chocolate sweetness scores and areal occupancy rate in the alpha frequency band were examined in the same way, revealing that there was no correlation between the chocolate sweetness score and areal occupancy rate in this frequency band $(n=18$; $R^{2}=0.038, F=1.66, P=0.22$; Fig. $3 b$ ). For the beta frequency band, again no correlation was evident between the chocolate sweetness scores and areal occupancy rate $(n=18$; $R^{2}=0.12, F=3.34, P=0.09$; Fig. $3 \mathrm{c}$ ). These results suggest that when taste perception was disturbed by unmatched olfactory stimulation, EEG activities in the theta frequency band were enhanced.

To test possibility that the increases in theta band EEG activity during unmatched olfactory stimulation were caused by the effect of the garlic odorant stimulation itself, we investigated the influences of odor stimulation on EEG frequency without taste stimulation. EEG signals were collected from nine healthy participants. Profiles of mean occupancy rates obtained from the frequency analysis under conditions of garlic and chocolate olfactory stimulation are shown in Fig. 4a, b, respectively. In Fig. 4c, profiles of the two conditions are superimposed. Figure $4 d$ shows the mean areal occupancy rate of the respective frequency bands under conditions of garlic and chocolate olfactory stimulation, respectively. To investigate whether patterns of EEG frequency bands between the two kinds of olfactory stimulation differed, we subtracted areal occupancy rates under the condition of garlic odor stimulation from those under the condition of chocolate odor stimulation and compared the results. Mean differences in theta, alpha and beta bands were $-0.92 \pm 3.15,-0.73 \pm 3.46$ and $-0.25 \pm 2.60$ (mean $\pm \mathrm{SD}$ ), respectively. One-way ANOVA performed using Origin8 software was used to evaluate the statistical significance of the areal difference values in the theta, alpha and beta frequency bands, respectively. No significant differences in areal difference values were apparent among these three frequency bands $(F=0.68 ; P=0.52)$, demonstrating 

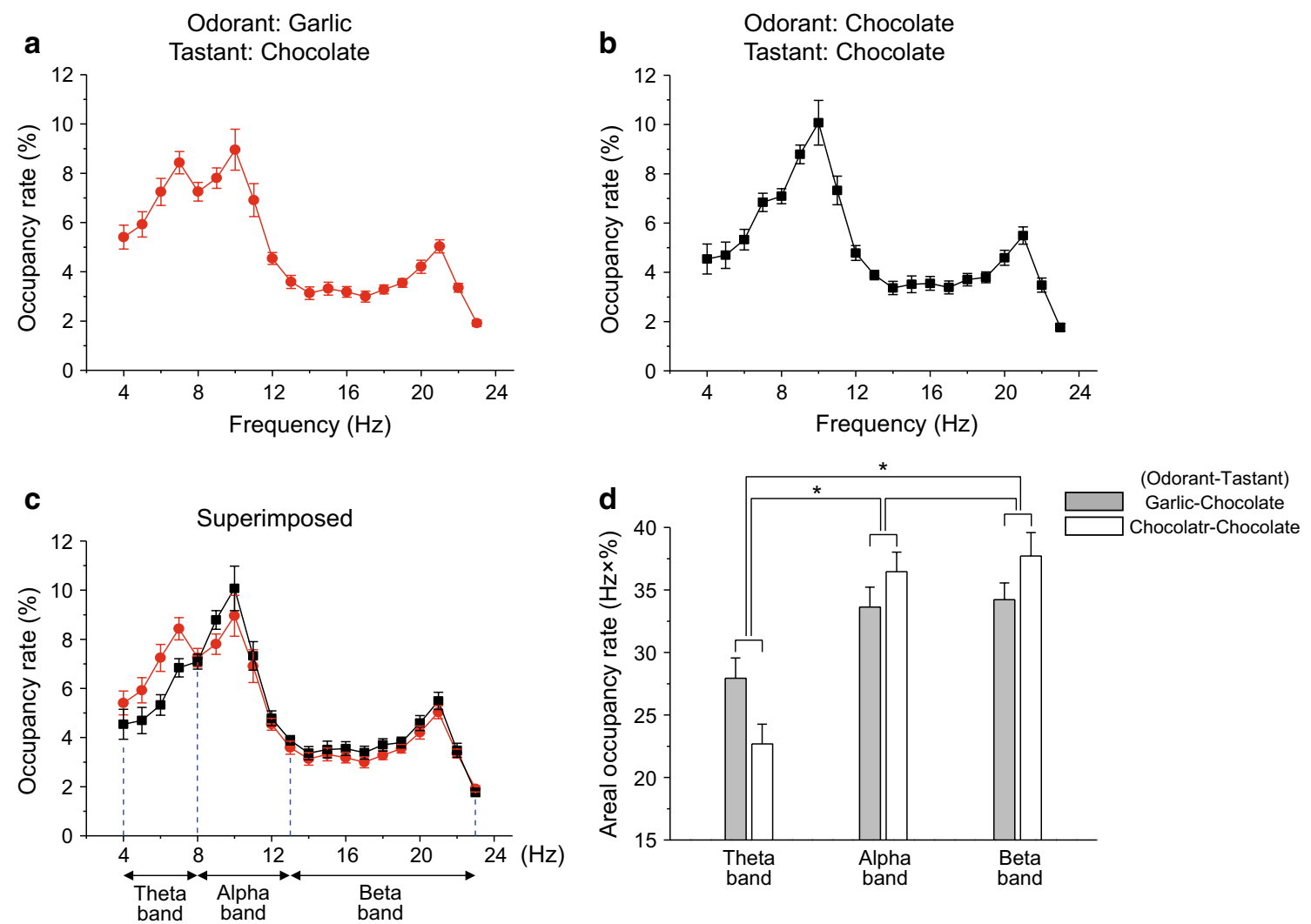

Fig. 2 Comparison of electroencephalogram (EEG) frequency activities obtained from unmatched and matched odor stimulation-evoked EEG responses, respectively, under the condition of tasting chocolate. a-c Profiles of mean occupancy rates obtained by Fast Fourier Transform (FFT) analysis of EEG signals. a, b Mean occupancy rate of the respective frequency bands $(4-23 \mathrm{~Hz})$ under conditions of unmatched (garlic) odor stimulation (a) and matched (chocolate) odor stimulation (b) while tasting chocolate (mean \pm standard error [SE]). c Pro-

that increases in theta band EEG activity in the case of unmatched olfactory stimulation were not attributable to the effect of the garlic odorant stimulation itself.

Profiles of mean occupancy rates obtained from frequency analysis in the case of chocolate taste stimulation without odor stimulation are shown in Fig. 5a. To investigate additional effects of smell on taste stimulation, mean areal occupancy rates of respective frequency bands under the condition of chocolate taste stimulation with and without odorant stimulation were compared (Fig. 5b, c). Areal occupancy rates under the condition of garlic odor stimulation + chocolate taste stimulation were subtracted from those under the condition of chocolate-taste stimulation'. Mean differences in theta, alpha and beta bands were $4.29 \pm 4.80$, $-2.49 \pm 3.13$ and $-2.74 \pm 5.13$ (mean $\pm \mathrm{SD})$, respectively. One-way ANOVA (Origin8) was used to evaluate the statistical significance of areal difference values in all three of these frequency bands, revealing significant differences $(F=7.25 ; P=0.003)$. Scheffe's post hoc test was then used files of mean occupancy rates for the two cases are superimposed, and the three frequency bands under study are shown (mean \pm SE). d Mean areal occupancy rates in the case of unmatched and matched odor stimulations are shown (mean $\pm \mathrm{SE}$ ). Differences in areal occupancy rates between cases are compared among the three frequency bands. Asterisk indicates that the difference in areal occupancy is significantly greater in the theta frequency band than in the other frequency bands (alpha, beta) under study

to detect significant differences between areal difference values in the respective frequency bands. These analyses revealed that differences in areal difference values between conditions of garlic odor stimulation + chocolate taste stimulation and chocolate taste stimulation were significantly greater in the theta frequency band than in other frequency bands (alpha: $F=5.2, P=0.01$; beta: $F=5.6, P=0.01$ ), demonstrating that an additional effect of garlic odor stimulation while tasting chocolate was enhancement of theta-band EEG activities. We then subtracted areal occupancy rates under the condition of chocolate-odor + chocolate taste stimulation conditions from those under the condition of chocolate taste stimulation. Mean differences in the theta, alpha and beta bands were $-0.97 \pm 3.35,0.34 \pm 2.75$ and $0.74 \pm 3.88$ (mean $\pm \mathrm{SD}$ ), respectively. One-way ANOVA (Origin8) was used to evaluate the significance of areal difference values in all three of these frequency bands, revealing that there were no significant differences in areal difference values among the three frequency bands $(F=0.64 ; P=0.54)$. This 

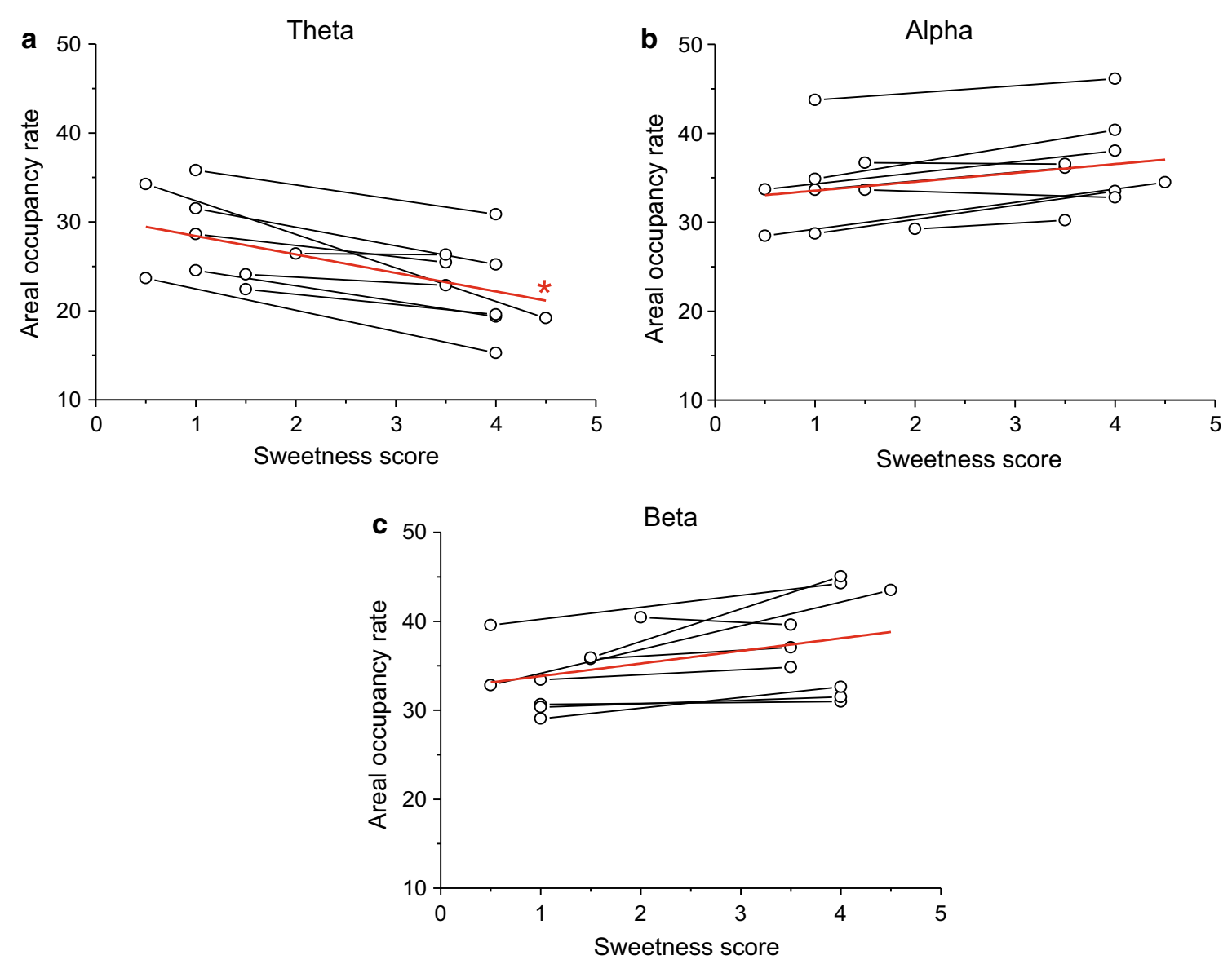

Fig. 3 Relationships between individual subjective sweetness scores and areal occupancy rate in the theta, alpha and beta frequency bands, respectively. Each data point represents the sweetness score and the areal occupancy rate. A set of two points was obtained from the same participant under the conditions of unmatched and matched odor

result shows that we could not find any additional effects of chocolate odor stimulation under the condition of tasting chocolate.

\section{Discussion}

Humans receive external information through various sensory receptors. This information is integrated during processes that range from perception to cognition and involve emotion and memory [8, 26]. Cognitive processes require past experiences, and humans can recognize objects when there is a stored experience of that object. One object contains various modes of information, and the specific combination of contained information is extremely important for recognition. When one mode of information does not match with the recollection of an object, recognition of that object may be difficult. During the cognitive process, the brain tries to cross-check unmatched information stimulation, respectively, and the set of two points was connected with a line. Data obtained from all participants are plotted together. Red lines show linear fitting. Asterisk denotes that in the theta frequency band sweetness scores correlate with areal occupancy rate

against the object, which may induce different patterns of brain activity compared with cases in which all information matches with the object.

In the study reported here, we used chocolate as a tastant and chocolate paste and garlic paste as odorants. All participants rated chocolate sweetness high when the matched odor stimulation condition was applied (Fig. 1). Under this condition, feelings that the food in the mouth definitely represented chocolate were increased, supporting the notion that the smell helped in the recognition of chocolate. On the other hand, all participants rated chocolate sweetness as low when the unmatched odor stimulation condition was applied. In this case, feelings that the food in the mouth was chocolate decreased, indicating that smell can disturb the recognition of chocolate. Although taste thresholds should be measured for more precise evaluation of taste perception, we used sweetness scores to allow an easy understanding of subjective feelings of chocolate taste, including deliciousness. 

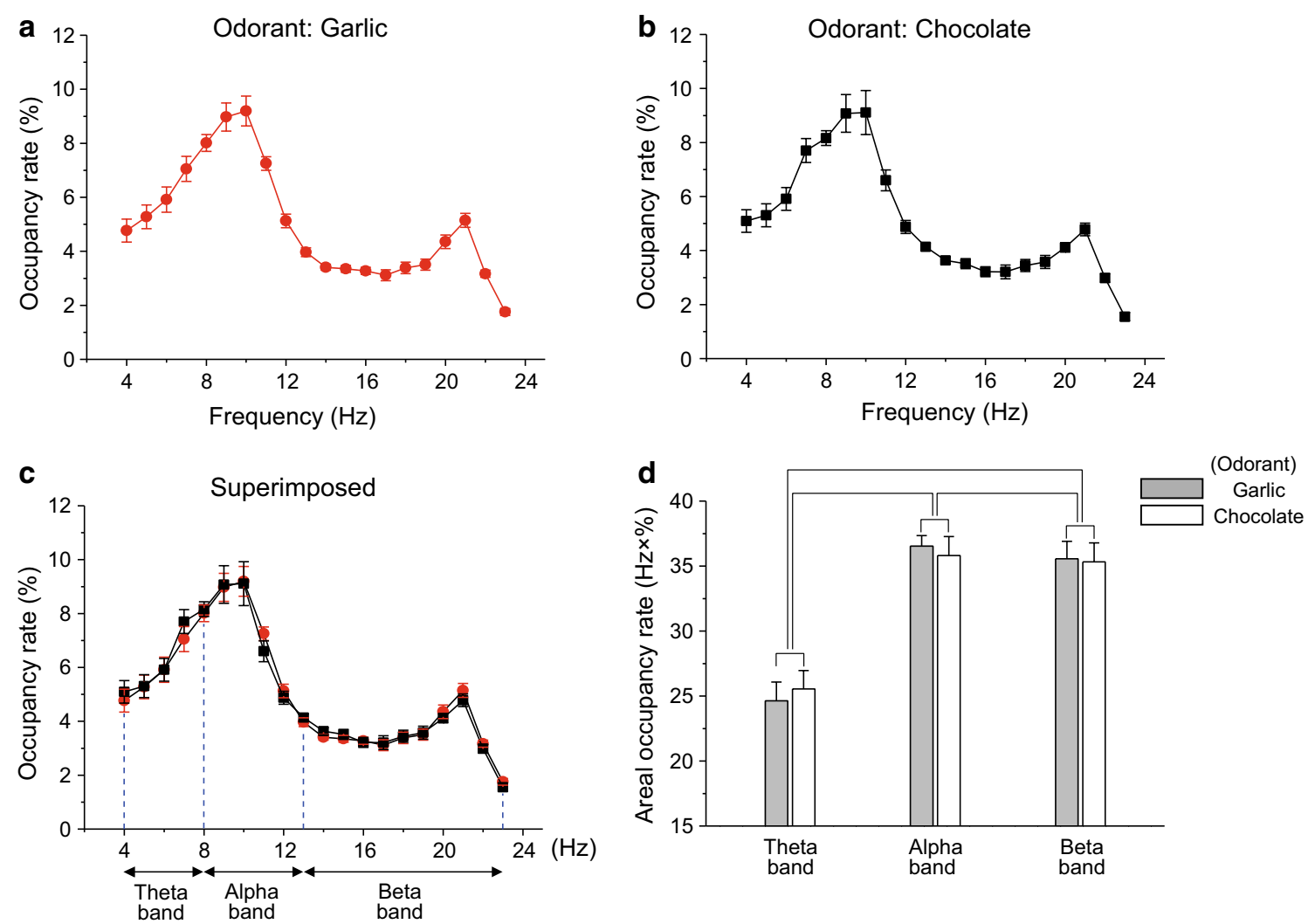

Fig. 4 Comparison of EEG frequency activities obtained from garlic and chocolate odor stimulation-evoked EEG responses without taste stimulation. a-c Profiles of mean occupancy rates obtained by FFT analysis of EEG signals (mean \pm SE). Mean occupancy rate of respective frequency $(4-23 \mathrm{~Hz})$ under conditions of garlic odor stimulation (a) and chocolate odor stimulation (b); c profiles of mean occupancy rates of the two conditions are superimposed, showing the

In our comparison of occupancy rate profiles between conditions of unmatched odor stimulation and matched odor stimulation while tasting chocolate, we identified a small bulge in the frequency range $4-8 \mathrm{~Hz}$ that we recognized as the theta rhythm in the case of unmatched odor stimulation (Fig. 2a). Given the large areal difference values in the theta frequency band, compared with those in other frequency bands, the theta frequency band may play a role in information processing when humans perceive smells that do not match the corresponding foods (Fig. 2). As the possibility that the small bulge in the theta frequency band was a garlic odor-evoked response had to be considered, we investigated profiles of occupancy rates under the condition of only odor stimulation (Fig. 4). However, we could find neither a small bulge in the theta frequency band nor differences between garlic odor-evoked and chocolate odor-evoked responses in all frequency bands (Fig. 4d). This result led us to conclude that the small bulge in the theta frequency band under the condition of unmatched odor stimulation and chocolate three frequency bands that we focused on. d Mean areal occupancy rates in the case of garlic and chocolate odor stimulation are shown. Differences in areal occupancy rates between the two cases are compared among the three frequency bands. Note that no significant differences in areal difference values are seen among the three frequency bands

tasting was unlikely to have been caused by only odor stimulation.

Figure 5a shows profiles of occupancy rate in the case of only chocolate taste stimulation without odorant stimulation. The profiles were similar to those under the condition of matched odor stimulation while tasting chocolate. Figure $5 \mathrm{c}$ shows no variance among differences between conditions of only chocolate tasting and matched odor stimulation while tasting chocolate in the respective frequency bands. We thus could not find additional effects of matched odor stimulation compared to taste stimulation. A possible cause of this result may be that the odor produced from chocolate itself in the oral cavity enters the nasal cavity and olfactory neurons are stimulated retronasally [22]. When retronasal odor stimulation is presented without orthnasal odor stimulation, retronasal olfaction might work more efficiently [27]. Figure $5 \mathrm{~b}$ shows a comparison of areal occupancy rates between the conditions of chocolate tasting alone and unmatched odor stimulation while tasting chocolate in the respective frequency bands. The statistical results were similar to the 


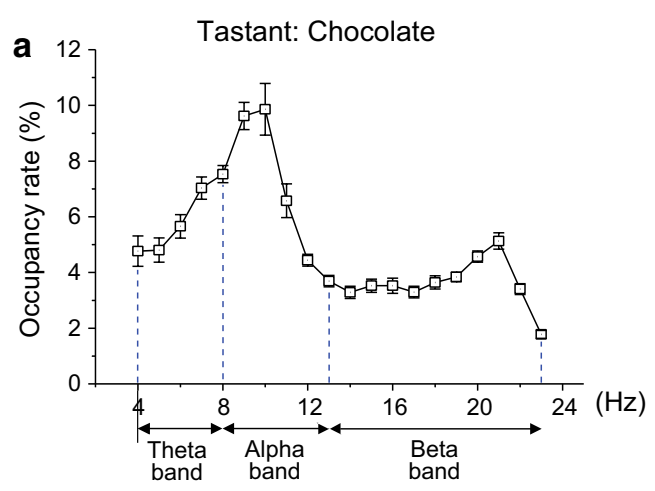

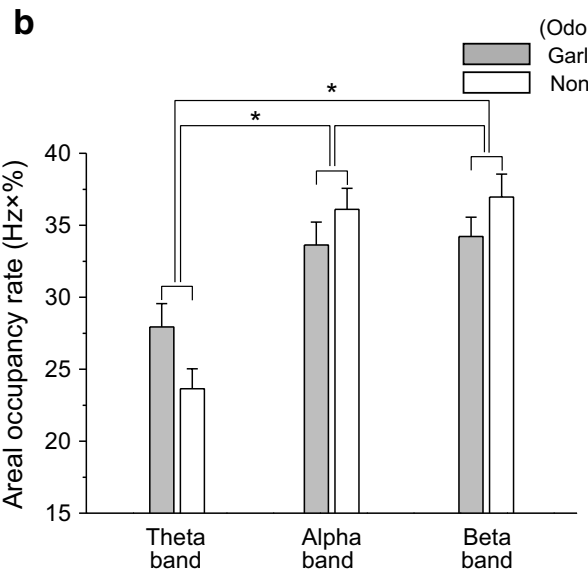

Fig. 5 Electroencephalogram frequency activities under the condition of chocolate taste stimulation and comparison of mean areal occupancy rates of the theta, alpha and beta frequency bands under conditions of no odor stimulation and odor stimulation, respectively, while tasting chocolate. a Mean occupancy rates for the theta, alpha and beta band frequencies $(4-23 \mathrm{~Hz})$, respectively, under the condition of no odor stimulation while tasting chocolate (mean \pm SE). b Mean areal occupancy rates under the conditions of garlic odor stimulation while tasting chocolate and no odor stimulation while tasting chocolate, respectively (mean \pm SE). Differences in areal occupancy rates

results obtained from the comparison between conditions of unmatched and matched odor stimulation while tasting chocolate (see Results and Fig. 2d). The similarity might be due to the very few additional effects of chocolate odor stimulation on EEG activities to chocolate taste stimulation, as mentioned above.

In recent years, frontal midline theta rhythm (Fm theta) in humans has been reported to reflect mental concentration as well as meditative state $[17,19,28-30]$. Human studies using EEG and magnetoencephalogram analyses suggest that the origin of Fm theta activities when executing tasks or making decisions that require mental concentration or a meditative state are interactions between the anterior cingulate cortex (ACC) and the medial prefrontal cortex (mPFC) [30-32]. Indeed, the ACC together with the orbitofrontal cortex are regarded as areas where taste and olfactory information converge [33]. Another candidate for sources of

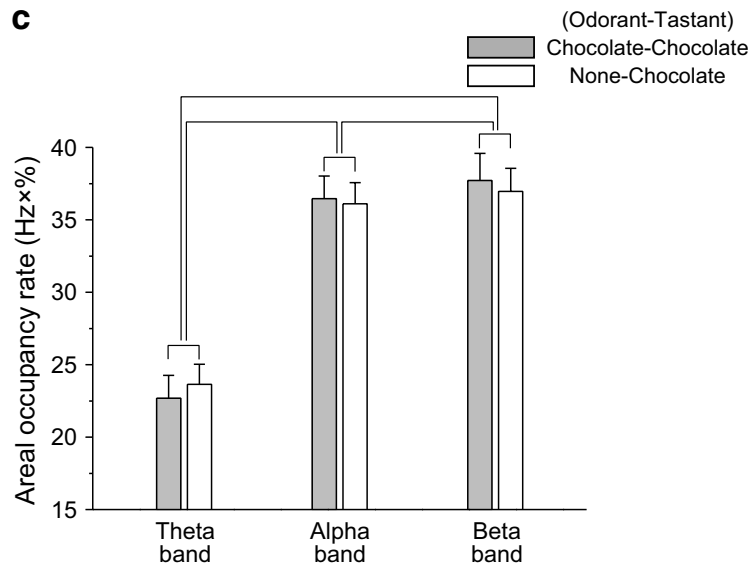

between the two conditions are compared among the theta, alpha and beta frequency bands. Asterisk indicates that the difference is significantly greater in the theta frequency band than in the other frequency bands. c Comparison of mean areal occupancy rates under conditions of chocolate odor stimulation while tasting chocolate and no odor stimulation while tasting chocolate (mean \pm SE). Differences in areal occupancy rates between the two conditions are compared among the theta, alpha and beta frequency bands. Note that there are no significant differences in areal difference values among the three frequency bands

Fm theta activities is the hippocampus. Recent studies suggest that oscillatory synchrony between the hippocampus and $\mathrm{mPFC}$ play a crucial role in not only memory integration, but also the retrieval of memories [34-36]. The hippocampus and $\mathrm{mPFC}$ are able to retrieve, exchange, integrate and re-encode multiple memories, as well as contribute to memory-based decision-making [36, 37]. An oscillatory coupling exists between the hippocampus and $\mathrm{mPFC}$, and theta oscillation is required when the association memory is transferred from the hippocampus to the mPFC [38-40].

The results of our study show that the theta band appeared while the participants were perceiving smells that did not match the food they were tasting. In addition, under this condition, the subjective feeling of sweetness decreased, and the participants tended to be unable to discern what the food was. The feeling of perturbance might induce concentration, resulting in the generation of the theta rhythm activity. 
Indeed, error-related theta waves have been observed in the mPFC [41]. Although we cannot address sources of the theta rhythm, our results suggest that theta-band brain activities emerge during the cross-checking of unmatched information.

Acknowledgements We wish to thank Dr. T. Akamatsu, T. Hasegawa, C. Yao, H. Y. Miyachi; Kanayama for experimental assistance. This study was partially supported by grants from the Ministry of Education, Culture, Sports, Science and Technology of Japan (Grant-in-Aid for Scientific Research C 22590966), and was partially supported by the Research Clusters Program of Tokushima University.

Author contribution HY contributed to the experimental design of the research, and drafted the manuscript. SM and HY performed experiments, and analyzed and interpreted the data.

\section{Compliance with ethical standards}

Conflict of interest The authors have no conflicts of interest to declare.

Ethical approval All procedures performed in studies involving human participants were in accordance with the ethical standards of the institutional and/or national research committee and with the 1964 Helsinki declaration and its later amendments or comparable ethical standards.

Informed consent Informed consent was obtained from all individual participants included in the study.

\section{References}

1. de Araujo IET, Rolls ET, Kringelbach ML, McGlone F, Phillips N (2003) Taste olfactory convergence, and the representation of the pleasantness of flavor, in the human brain. Eur J Neurosci 18:2059-2068

2. Rolls ET (2005) Taste, olfactory, and food texture processing in the brain, and the control of food intake. Physiol Behav 85:45-56

3. Lemon CH, Katz DB (2007) The neural processing of taste. BMC Neurosci 8[Suppl 3]:S5

4. Wilson DA, Sullivan RM (2011) Cortical processing of odor objects. Neuron 72:506-519

5. Soudry Y, Lemogne C, Malinvaud D, Consoli SM, Bonfils P (2011) Olfactory system and emotion: common substrates. Eur Ann Otorhinolaryngol Head Neck Dis 128:18-23

6. Kadohisa M (2013) Effects of odor on emotion, with implications. Front Syst Neurosci 7:1-6 (Article 66)

7. LeDoux JE (2000) Emotion circuits in the brain. Annu Rev Neurosci 23:155-184

8. Dolan RJ (2002) Emotion, cognition, and behavior. Science 298:1191-1194

9. Mériau K, Isabell Wartenburger I, Kazzer P, Prehn K, Lammers $\mathrm{CH}$, van der Meer E, Villringer A, Heekerena HR (2006) A neural network reflecting individual differences in cognitive processing of emotions during perceptual decision making. NeuroImage 33:1016-1027

10. Djordjevic J, Zatorre RJ, Jones-Gotman M (2004) Odor-induced changes in taste perception. Exp Brain Res 159:405-408

11. Prescott J, Johnstone V, Francis J (2004) Odor-taste interactions: effects of attentional strategies during exposure. Chem Senses 29:331-340

12. Small DM, Prescott J (2005) Odor/taste integration and the perception of flavor. Exp Brain Res 166:345-357
13. Spence C (2015) Multisensory flavor perception. Cell 161:24-35

14. Hornung DE, Enns MP (1987) Odor-taste mixtures. Ann N Y Acad Sci 510:86-90

15. Macht M, Dettmer D (2006) Everyday mood and emotions after eating a chocolate bar or an apple. Appetite 46:332-336

16. Meier BP, Noll SW, Molokwu OJ (2017) The sweet life: the effect of mindful chocolate consumption on mood. Appetite 108:21-27

17. Inanaga K (1998) Fontal midline theta rhythm and mental activity. Psychiatry Clin Neurosci 52:555-566

18. Klimesch W, Freunberger R, Sauseng P, Gruber W (2008) A short review of slow phase synchronization and memory: evidence for control processes in different memory systems? Brain Res 1235:31-44

19. Mitchell DJ, McNaughton N, Flanagan D, Kirk IJ (2008) Frontalmidline theta from the perspective of hippocampal 'theta'. Prog Neurobiol 86:156-185

20. Yoshimura H, Morimoto S, Okuro M, Segami N, Kato N (2010) Evaluations of dementia by EEG frequency analysis and psychological examination. J Physiol Sci 60:383-388

21. Başar E (2012) A review of alpha activity in integrative brain function: Fundamental physiology, sensory coding, cognition and pathology. Int J Psychophysiol 86:1-24

22. Bojanowski V, Hummel T (2012) Retronasal perception of odors. Physiol Behav 107:484-487

23. Voirol E, Daget N (1986) Comparative study of nasal and retronasal olfactory perception. Food Sci Technol 19:316-319

24. Heilmann S, Hummel T (2004) A new method for comparing orthonasal and retronasal olfaction. Behav Neurosci 118:412-419

25. Yoshimura H, Honjo M, Sugai T, Kawabe M, Kaneyama K, Segami N, Kato N (2011) Influences of audio-visual environments on feelings of deliciousness during having sweet foods: an electroencephalogram frequency analysis study. Nutritional Neurosci $14: 210-215$

26. LaBar KS, Cabeza R (2006) Cognitive neuroscience of emotional memory. Nature Rev Neurosci 7:54-64

27. Welge-Lüssen A, Husner A, Wolfensberger M, Hummel T (2009) Influence of simultaneous gustatory stimuli on orthonasal and retronasal olfaction. Neurosci Lett 454:124-128

28. Sasaki K, Nambu A, Tsujimoto T, Matsuzaki R, Kyuhou S, Gemba H (1996a) Studies on integrative functions of the human frontal association cortex with MEG. Cogn Brain Res 5:165-174

29. Sasaki K, Tsujimoto T, Nishikawa S, Nishitani N, Ishihara T (1996b) Frontal mental theta wave recorded simultaneously with magnetoencephalography and electroencephalography. Neurosci Res 26:79-81

30. Gevins A, Smith ME, McEvoy L, Yu D (1997) High-resolution EEG mapping of cortical activation related to working memory: effects of task difficulty, type of processing, and practice. Cereb Cortex 7:374-385

31. Asada H, Fukuda Y, Tsunoda S, Yamaguchi M, Tonoike M (1999) Frontal midline theta rhythms reflect alternative activation of prefrontal cortex and anterior cingulate cortex in humans. Neurosci Lett 274:29-32

32. Kubota Y, Sato W, Toichi M, Murai T, Okada T, Hayashi A, Sengoku A (2001) Frontal midline theta rhythm is correlated with cardiac autonomic activities during the performance of an attention demanding meditation procedure. Cogn Brain Res 11:281-287

33. Small DM, Voss J, Mak YE, Simmons KB, Parrish T, Gitelman D (2004) Experience-dependent neural integration of taste and smell in the human brain. J Neurophysiol 92:1892-1903

34. Kaplan R, Bush D, Bonnefond M, Bandettini PA, Barnes GR, Doeller CF, Burgess N (2014) Medial prefrontal theta phase coupling during spatial memory retrieval. Hippocampus 24:656-665

35. Trimper JB, Stefanescu RA, Manns JR (2014) Recognition memory and theta-gamma interactions in the hippocampus. Hippocampus 24:341-353 
36. Backus AR, Schoffelen JM, Szebényi S, Hanslmayr S, Doeller CF (2016) Hippocampal-prefrontal theta oscillations support memory integration. Curr Biol 26:450-457

37. Gordon JA (2011) Oscillations and hippocampal-prefrontal synchrony. Curr Opin Neurobiol 21:486-491

38. Stella F, Treves A (2011) Associative memory storage and retrieval: involvement of theta oscillations in hippocampal information processing. Neural Plast 2011:1-15

39. Siapas AG, Lubenov EV, Wilson MA (2005) Prefrontal phase locking to hippocampal theta oscillations. Neuron 46:141-151

40. Igarashi KM (2015) Plasticity in oscillatory coupling between hippocampus and cortex. Curr Opin Neurobiol 35:163-168
41. Cohen MX (2011) Error-related medial frontal theta activity predicts cingulate-related structural connectivity. NeuroImage 55:1373-1383

Publisher's Note Springer Nature remains neutral with regard to jurisdictional claims in published maps and institutional affiliations. 